\title{
PERFIL LIPÍDICO E COMPOSIÇÄO CORPORAL NA DEFICIÊNCIA DO HORMÔNIO DE CRESCIMENTO EM ADULTOS
}

\author{
Priscila M. Lobo*, flávia l. Conceição, Débora V. Soares, luciana D. C. Spina, Rosane R. L. O. Brasil, Eliana M. C. da Silva, Mário Vaisman
}

Estudo realizado no Hospital Universitário Clementino Fraga Filho, Rio de Janeiro, RJ.

* Correspondência:

Av. Tenente Coronel Muniz de Aragão, 898, bl. 1 /

1107, Rio de Janeiro, RJ,

Cep 22765-001.

priscilobo@aol.com

\begin{abstract}
RESUMO
OвJEтrvo. A síndrome da deficiência de hormônio de crescimento (DGH) no adulto e o conseqüente aumento no risco cardiovascular têm sido bastante estudados nos últimos anos. De grande relevância clínica são as alterações na composição corporal com aumento do tecido adiposo visceral e perfil lipídico adverso.

Métodos. Estudou-se o perfil lipídico e o tecido adiposo visceral de 31 adultos com DGH comparado com um grupo controle de indivíduos saudáveis pareados por idade, sexo e índice de massa corporal (IMC). A avaliação da gordura visceral foi feita por tomografia computadorizada de abdome e por medidas antropométricas, através do IMC $\left(\mathrm{Kg} / \mathrm{m}^{2}\right)$ e da medida da cintura (cm). A avaliação do perfil lipídico foi obtida através de dosagens laboratoriais de colesterol (CT), triglicerídeos (TG), HDL, LDL, apolipoproteínas A e B e lipoproteína (a).
\end{abstract}

Resultados. Foi observado aumento do tecido adiposo visceral nos pacientes DGH (|156,66 $\pm 72,72$ vs. || $13,5 \mid \pm 32,97$ $\mathrm{cm}^{2}$; p-valor $\left.=0,049\right)$, além de aumento nos níveis de TG $(158,80 \pm 80,29 \mathrm{vs}$. $97,17 \pm 12,37 \mathrm{mg} / \mathrm{dl}$; p-valor $=0,007) \mathrm{e}$ diminuição nos níveis de $\operatorname{HDL}(45,4 \mid \pm 13,30$ vs. $55,34 \pm 14,31 \mathrm{mg} / \mathrm{d}$; $p$-valor $=0,002)$. Não houve diferença entre os demais parâmetros do perfil lipídico e nas medidas antropométricas.

Conclusão. Adultos deficientes de GH apresentam aumento da adiposidade visceral e aumento das concentrações de TG com diminuição das concentrações de HDL.

Unitermos: Lipídios-metabolismo. Composição corporal. Somatropina-deficiência. Humano. Adulto.

\section{INTRODUÇÃO}

O uso de hormônio de crescimento $(G H)$ apresenta indicação clínica bem definida em crianças com deficiência de $\mathrm{GH}$, a fim de promover o crescimento linear há cerca de 40 anos. Já a terapia de reposição para adultos deficientes só começou a ser realizada no fim da década de 80, quando estudos clínicos pioneiros demonstraram seus efeitos benéficos, tornando a síndrome de deficiência de GH (SDGH) bem caracterizadal,2.

Desde então, suspeitava-se que tais pacientes, quando não tratados, apresentavam fatores de risco que contribuiriam para o aumento na incidência de doenças cardiovasculares ${ }^{3} \mathrm{e}$ aumento na mortalidade cérebro-vascular4.

Esses fatores de risco seriam as alterações na composição corporal, caracterizadas pela diminuição de massa magra e aumento de massa gorda com acúmulo de gordura visceral ${ }^{5}$, além de alterações nos metabolismos lipídico (Quadro I) e glicídico, com diminuição da sensibilidade à insulina ${ }^{7,8,9}$. Essas anormalidades tornam bastante similares a SDGH em adultos e a síndrome plurimetabólica, que se caracteriza por diversos sinais e sintomas clínicos, principalmente obesidade visceral e abdominal, resistência insulínica, dislipidemia e aterosclerose prematura.

Por isso, torna-se importante a avaliação do metabolismo lipídico e da adiposidade visceral em adultos com DGH comparados a um grupo controle adequado.

\section{Objetivos}

Em um grupo de adultos com deficiência de hormônio de crescimento, comparado a um grupo controle pareado por idade, sexo e índice de massa corporal, objetivamos avaliar:

I) O perfil lipídico, através das dosagens de colesterol total e frações, triglicerídeos e apolipoproteínas.

2) A composição corporal através de medidas antropométricas e tomografia computadorizada de abdome.

\section{Métodos \\ Casuística}

Foram avaliados 31 pacientes deficientes de hormônio de crescimento, 19 mulheres e 12 homens, com idade entre 20 e 61 anos (média de 4I,83 anos), e 29 indivíduos saudáveis (controles) pareados por sexo, idade e índice de massa corporal (IMC). Todos os pacientes apresentavam deficiência de hormônio do crescimento por mais de um ano. Seis com deficiência desde a infância, com diagnóstico de hipopituitarismo idiopático e 25 com hipopituitarismo adquirido durante a vida adulta. Destes, 13 pacientes apresentavam hipopituitarismo pós-cirúrgico e/ou pós radioterapia hipofisária; um, histiocitose X; e II mulheres apresentavam diagnóstico de síndrome de Sheehan (necrose hipofisária pós-parto). Um com déficit isolado e 30 com mais de uma deficiência hormonal. Foram incluídos os pacientes que apresentaram pico de 
LOBO PM ET AL.

\begin{tabular}{|c|c|c|c|c|c|c|}
\hline \multicolumn{7}{|c|}{ Quadro I - Perfil lipídico em adultos com DGH } \\
\hline Autor & Ano & $\mathrm{n}$ & $\underline{\text { CT }}$ & $\underline{\text { TG }}$ & $\underline{\mathrm{HDL}}$ & $\underline{\mathbf{L D L}}$ \\
\hline Rosen & 1993 & 104 & $\leftrightarrow$ & $\uparrow$ & $\downarrow$ & \\
\hline Cuneo & 1993 & 24 & $\uparrow$ & $\uparrow$ & $\downarrow$ & $\uparrow$ \\
\hline de Bôer & 1994 & 64 & $\uparrow$ & $\leftrightarrow$ & $\leftrightarrow$ & $\uparrow$ \\
\hline Hew & 1996 & 30 & $\leftrightarrow$ & $\uparrow$ & $\downarrow$ & $\leftrightarrow$ \\
\hline O'Neal & 1996 & 30 & $\leftrightarrow$ & $\uparrow^{1 *}$ & $\downarrow$ & $\leftrightarrow$ \\
\hline Attanasio & 1997 & 173 & $\uparrow$ & & $\downarrow$ & $\uparrow$ \\
\hline AlShoumer & 1997 & 67 & $\uparrow$ & $\uparrow$ & $\leftrightarrow$ & $\uparrow$ \\
\hline Lobo & 2003 & 31 & $\leftrightarrow$ & $\uparrow$ & $\downarrow$ & $\leftrightarrow$ \\
\hline
\end{tabular}

n- número de pacientes; CT- colesterol total; TG- triglicerídeos; HDL-C- HDL-colesterol;

LDL-C- LDL-colestero;; $\uparrow$ - aumento significativamente estatístico; $\downarrow$ - diminuiç̧ão significativamente estatística; $\leftrightarrow$ - ausência de significância estatistica; $\uparrow^{*}$ - aumento sem significância estatística

$\mathrm{GH}$ menor que $3 \mathrm{ng} / \mathrm{ml}$ em pelo menos dois testes de estímulo farmacológicos: a) Teste de tolerância à insulina ( 0 , I - 0, I 5 UI de insulina regular/Kg de peso, endovenosa). Todos manifestaram sinais/sintomas de hipoglicemia e/ ou níveis de glicemia com queda de $50 \%$ em relação ao basal ou menor que $40 \mathrm{mg} / \mathrm{dl}$. b) Teste do glucagon (I mg, intra-muscular $)^{10}$.

Os pacientes estavam em terapia de reposição com tiroxina, glicocorticóides e esteróides sexuais com doses estáveis por pelo menos três meses antes de iniciar o estudo e os respectivos eixos estavam normofuncionantes.

Como critérios de exclusão adotamos: presença de doença mental, gravidez, diabetes mellitus, hipertensão arterial descompensada (pressão arterial sistólica acima de 160 e/ ou diastólica acima de $90 \mathrm{mmHg}$ ), uso de $\mathrm{GH}$ nos últimos 12 meses, doença aguda severa, doença hepática ou renal crônica, história de malignidade e acromegalia prévia.

Os controles foram selecionados entre os parentes e amigos dos pacientes e funcionários do hospital, sendo que as mulheres em menopausa estavam obrigatoriamente em uso de terapia de reposição hormonal.

Os objetivos e métodos do estudo foram explicados aos pacientes e controles e todos assinaram um termo de consentimento livre e esclarecido, aprovado pelo Comitê de Ética em Pesquisa do HUCFF e da Faculdade de Medicina da UFRJ.

\section{Metodologia}

Os pacientes e os controles foram avaliados ambulatorialmente, com entrevista e exame físico completo, registrando-se dados como: idade, sexo, cor, diagnóstico etiológico, tempo de evolução de doença, deficiências hormonais associadas e as respectivas reposições, outras doenças associadas e uso de medicações (respeitando-se os critérios de exclusão do estudo), ressaltando diagnóstico prévio de hipertensão arterial sistêmica, dislipidemia, tabagismo e história familiar de diabetes mellitus tipo $2 \mathrm{em}$ parentes de primeiro grau. Após a primeira entrevista ambulatorial, os pacientes foram submetidos à confirmação da deficiência de $\mathrm{GH}$ através dos testes de tolerância à insulina e glucagon, e em seguida encaminhados para avaliação do perfil lipídico.
O perfil lipídico - colesterol (CT), HDL, LDL, triglicerídeos (TG), lipoproteína (a) (Lp (a)), apolipoproteína A (Apo A) e apolipoproteína $B$ (Apo B) - foi avaliado em todos os pacientes e controles após jejum noturno de I 2 horas assim como dosagem de glicose, T4 livre, estradiol ou testosterona.

Análise bioquímica: GH: dosado através de ensaio imunométrico por quimioluminescência IMMULITE-DPC, United Kingdon (valor de referência de 0,06 a 5,0 ng/ml). Colesterol total: dosado através de método enzimático, Colestatenzimático- Wiener lab. (médias de valores entre 157 e $47 \mid \mathrm{mg} / \mathrm{dl}$ ). HDL: avaliado por método de inibição seletiva, HDL LE - LABTEST (médias de valores entre 33,I e 72,3). Triglicerídeo: avaliado através de método enzimático Ecoline 25 - Merck (médias de valores entre 41,8 e 368 $\mathrm{mg} / \mathrm{dl})$. Apo A e Apo B: dosadas através de reação imunoquímica avaliada por nefelometria, $\mathrm{N}$ anti- soros para apolipoproteína A- I e apolipoproteína B humanas - DADE BEHRING (valores de referência para Apo A-I de I 10 a 215 mg/dl e para Apo B de 55 a 140 mg/dl). Lp (a): determinação quantitativa mediante nefelometria cinética, LPA-Beckman (valor de referência para normalidade de $30 \mathrm{mg} / \mathrm{dl}$ ). $L D L$ : calculado pela fórmula de Friedwald: $L D L=C T-[H D L+$ $(\mathrm{TG} / 5)]$. As demais dosagens bioquímicas foram feitas por métodos automatizados pela rotina do laboratório de análises clínicas do HUCFF.

Nessa ocasião, os pacientes e controles foram pesados com roupas leves, sem sapatos, em balança mecânica Filizola e medidos com estadiômetro, assim como foi avaliada a menor circunferência abdominal com fita métrica não elástica. Essas medidas foram realizadas sempre na mesma balança e pelo mesmo examinador. O IMC foi calculado de acordo com a fórmula peso (Kg) / altura (m ao quadrado).

Todos os pacientes foram encaminhados para realização de tomografia helicoidal de abdome, sem contraste oral ou venoso, para quantificação da gordura visceral e subcutânea. $O$ equipamento utilizado foi o modelo PRO-SPEED, da marca GE. Todos os exames foram realizados pelo mesmo avaliador. Para avaliação da gordura abdominal total, visceral e subcutânea realizamos pequena hélice de quatro cortes, com $10 \mathrm{~mm}$ de espessura e espaçamento. As medidas foram feitas selecionando-se a imagem abdominal de maior diâmetro. Contornamos com o cursor a circunferência abdominal e medimos todas as densidades, em especial as de tecido gorduroso (área total de gordura abdominal); em seguida, contornamos somente o conteúdo visceral e repetimos o procedimento. Dessa forma, limitamos o contorno da parede abdominal e adquirimos os valores das áreas de gordura total, visceral e subcutânea.

Para comparar níveis de lipídios séricos, gordura visceral, idade e IMC entre grupo controle e pacientes e entre pacientes do sexo feminino e masculino no tempo basal foi utilizado teste t de Student não pareado para variáveis com distribuição homogênea e o teste não paramétrico de Mann-Whitney para variáveis com distribuição não homogênea. Para avaliação das correlações entre as variáveis estudadas foi utilizado o coeficiente de Pearson. $O$ nível de significância estatística foi de 5\% ( $p<0,05)$. 


\begin{tabular}{|c|c|c|c|}
\hline \multicolumn{4}{|c|}{ Tabela I - Metabolismo lipídico e medidas antropométricas } \\
\hline & $\begin{array}{l}\text { Pacientes } \\
(n=31)\end{array}$ & $\begin{array}{l}\text { Controles } \\
(n=29)\end{array}$ & p-valor \\
\hline Idade (anos) & $\overline{41,83( \pm 10,57)}$ & $39,17( \pm 9,49)$ & 0,30 \\
\hline Peso (Kg) & $68,14( \pm 16)$ & $71,82( \pm 9,12)$ & 0,28 \\
\hline Cintura $(\mathrm{cm})$ & $85,25( \pm 12,11)$ & $85,27( \pm 8,52)$ & 0,99 \\
\hline $\operatorname{IMC}\left(\mathrm{Kg} / \mathrm{m}^{2}\right)$ & $26,91( \pm 5,43)$ & $26,63( \pm 3,74)$ & 0,82 \\
\hline Colesteroltotal (mg/dl) & $195,09( \pm 33,45)$ & $202,17( \pm 49,51)$ & 0,51 \\
\hline $\mathrm{LDL}(\mathrm{mg} / \mathrm{dl})$ & $115,44( \pm 27,76)$ & $124,34( \pm 47,19)$ & 0,37 \\
\hline $\mathrm{HDL}(\mathrm{mg} / \mathrm{dl})$ & $45,4 \mid( \pm \mid 3,30)$ & $55,34( \pm \mid 4,13)$ & 0,007 \\
\hline Triglicerídeo (mg/dl) & $158,80( \pm 80,29)$ & $97,17( \pm 62,37)$ & 0,002 \\
\hline Apolipoproteína A(mg/dl) & $137,67( \pm 36,70)$ & $140,24( \pm 26,44)$ & 0,75 \\
\hline Apolipoproteína B(mg/dl) & $111,32( \pm 27,60)$ & $103,58( \pm 30,77)$ & 0,30 \\
\hline Lipoproteína a(mg/dl) & $42,76( \pm 34,90)$ & $40,64( \pm 39,17)$ & 0,82 \\
\hline
\end{tabular}

\begin{tabular}{|c|c|c|c|}
\hline \multicolumn{4}{|c|}{ Tabela 2 - Níveis de TG e HDL nas mulheres e homens } \\
\hline & \multicolumn{3}{|c|}{ Feminino } \\
\hline & Pacientes & Controles & p valor \\
\hline TG & $|47,68 \pm 68,5|$ & $88,31 \pm 35,91$ & 0,004 \\
\hline \multirow[t]{3}{*}{ HDL } & $48,57 \pm 13,16$ & $59,75 \pm|4,4|$ & 0,022 \\
\hline & \multicolumn{3}{|c|}{ Masculino } \\
\hline & Pacientes & Controles & p valor \\
\hline TG & $|76,4| \pm 96,69$ & $108,07 \pm 85,04$ & 0,073 \\
\hline HDL & $40,41 \pm 12,43$ & $49,92 \pm 12,18$ & 0,066 \\
\hline
\end{tabular}

\begin{tabular}{lcc}
\hline \multicolumn{3}{c}{ Tabela 3 - Tecido adiposo visceral $\left(\mathrm{cm}^{2}\right)$} \\
\hline Estatísticas descritivas & $\frac{\text { Pacientes }}{29}$ & Controles \\
Indivíduos (n) & 156,66 & 13 \\
Média & 72,72 & $|13,5|$ \\
Desvio padrão & & 0,049 \\
p valor & & 32,97 \\
\hline
\end{tabular}

Tabela 4 - Tecido adiposo visceral $\left(\mathrm{cm}^{2}\right)$ - mulheres $\mathrm{x}$ homens

\begin{tabular}{|c|c|c|c|c|}
\hline & \multicolumn{2}{|c|}{ Feminino } & \multicolumn{2}{|c|}{ Masculino } \\
\hline & Pacientes & Controles & Pacientes & Controles \\
\hline $\begin{array}{l}\text { Gordura } \\
\text { visceral }\end{array}$ & $150,06 \pm 55,26$ & $106,65 \pm 21,66$ & $|65,45 \pm 93| \mid$, & $124,48 \pm 46,86$ \\
\hline p valor & 0,0 & & & 0,37 \\
\hline
\end{tabular}

Tabela 5 - Coeficiente de correlação entre as variáveis estudadas

\begin{tabular}{lcc}
\hline Correlações & $\frac{\mathbf{R}}{\mathrm{p} \text {-valor }}$ \\
\cline { 2 - 3 } Gordura visceral vs. TG & 0,352 & 0,024 \\
Gordura visceral vs. HDL & $-0,123$ & 0,443 \\
Gordura visceral vs. cintura & 0,52 & 0,000 \\
TG vs. HDL & $-0,28$ & 0,03 \\
Gordura visceral vs. TG* & 0,531 & 0,008 \\
Gordura visceral vs. TG*** & 0,241 & 0,352 \\
\hline *mulheres ** homens & &
\end{tabular}

\section{Resultados}

\section{Metabolismo lipídico}

Não houve diferença significativa entre grupo controle e pacientes em relação a CT, LDL, Lp(a), Apo A e Apo B. TG foi mais elevado ( $p=0,002)$ e HDL menor ( $p=0,007)$ nos DGH (Tabela I).

Quando separamos por sexo, encontramos significância estatística somente nas mulheres (Tabela 2).

\section{Medidas antropométricas e gordura visceral}

Os pacientes foram pareados com os controles por IMC e apresentaram médias semelhantes de cintura (Tabela I). A medida do tecido adiposo visceral, avaliada em 29 pacientes e 13 controles, foi significativamente maior nos pacientes (Tabela 3). Ao subdividimos por sexo, encontramos significância estatística apenas nas mulheres (Tabela 4).

\section{Correlação gordura visceral vs. metabolismo lipídico}

Houve correlação positiva entre a medida de gordura visceral e níveis de TG, e gordura visceral e medida da cintura tanto nos pacientes quanto nos controles, assim como a correlação negativa entre níveis de TG e HDL. Não encontramos correlação negativa entre gordura visceral e níveis de HDL. Quando subdividimos por sexo, encontramos correlação positiva entre gordura visceral e TG apenas nas mulheres (Tabela 5).

\section{Discussão}

A idade média dos pacientes (4I anos) foi semelhante à maioria dos trabalhos publicados com os quais comparamos nossos dados. Os pacientes avaliados apresentavam sobrepeso (IMC de 26,9l Kg/ $\mathrm{m}^{2}$ ), com médias semelhantes de cintura em relação aos controles. Além disso, apresentaram aumento da adiposidade visceral, avaliada pela tomografia computadorizada de abdome, caracterizando uma distribuição anormal de gordura corporal com predomínio central. Esses achados estão de acordo com a literatura ${ }^{8,11-14}$.

Os níveis de triglicerídeos encontrados nos pacientes foram maiores que nos controles e os níveis de HDL foram menores, achados consistentes com outros estudos ${ }^{2,15,16}$.

Rosen et al. ${ }^{2}$ foram os primeiros a estudar o perfil lipídico em 104 pacientes com deficiência de GH adquirida na vida adulta 
LoBO PM ET AL.

comparados com controles saudáveis. Os pacientes apresentavam níveis maiores de TG e menores de HDL que os controles.

Com relação aos níveis de colesterol total, LDL, Apo A, Apo B e Lp (a) não encontramos diferença entre pacientes e controles. Tais achados diferem das alterações encontradas por Boer et al. ${ }^{17}$, que avaliaram 64 adultos do sexo masculino com DGH adquirida na infância comparados com um grupo controle pareado por idade e sexo, e verificaram níveis maiores de colesterol total e LDL nos pacientes, porém com níveis semelhantes de triglicerídeos e HDL.

Em 1997, Attanasio et al. ${ }^{18}$ estudaram 173 deficientes de GH comparados com indivíduos normais e também encontraram níveis maiores de colesterol total e LDL, assim como níveis menores de HDL. Neste estudo, foram avaliados pacientes com deficiência de DGH adquirida na vida adulta $(n=99)$ e pacientes deficientes desde a infância $(n=74)$. Níveis de CT foram elevados em ambos os grupos, quando comparados com indivíduos normais. Os níveis de HDL foram menores nos pacientes com DGH adquirida na idade adulta do que nos indivíduos normais. Tal fato não ocorreu nos pacientes com DGH adquirida na infância, embora $72 \%$ dos pacientes com DGH adquirida na vida adulta e 54\% dos DGH desde a infância apresentassem concentrações alteradas de HDL.

O'Neal et al. ${ }^{19}$ compararam 30 adultos hipopituitários em reposição hormonal convencional com dois grupos controles. O primeiro era composto por indivíduos pareados para sexo, idade e IMC, e o segundo, por indivíduos pareados para sexo e idade, porém com IMC representativo da população geral. Foram encontrados níveis diminuídos de HDL nos pacientes hipopituitários, assim como níveis aumentados de TG, apesar de não haver significância estatística neste último achado. Não houve diferença estatisticamente significativa nos níveis de CT, LDL, Lp(a) ou Apo B. Diferenças nas concentrações de Apo A foram consistentes com os níveis de HDL. Os pacientes apresentaram os menores níveis de Apo A quando comparados com os dois grupos. Neste estudo, também foi demonstrado que o tamanho das partículas de LDL foi significativamente menor nos pacientes. $O$ achado das partículas pequenas e densas de LDL está associado ao aumento de três vezes do risco para ocorrência de infarto agudo do miocárdio ${ }^{20}$. Além disso, essas partículas estão associadas a um perfil lipídico aterogênico em associação com hipertrigliceridemia e diminuição de $\mathrm{HDL}^{21}$.

Cuneo et al. ${ }^{22}$, avaliando 24 adultos com DGH pareados com controles para idade, peso e sexo, demonstraram níveis maiores de $C T$, LDL, Apo B e TG nos pacientes, bem como concentrações menores de HDL do que nos controles.

Al Shoumer et al. ${ }^{23}$ estudaram 67 deficientes de $\mathrm{GH}$ e 87 controles normais e encontraram concentrações de TG, CT, LDL e Apo B significativamente maiores nos pacientes que nos controles. Ambos apresentavam concentrações semelhantes de HDL, Apo A e Lp (a).

Esses achados sugerem que adultos com deficiência de GH apresentam um perfil lipídico adverso que está relacionado à aterosclerose prematura. O resumo destes trabalhos encontra-se no Quadro I.

Quando subdividimos por sexo e analisamos o grupo de pacientes mulheres vs. controles mulheres e o grupo de pacientes homens vs. controles homens, observamos que as alterações encontradas (níveis maiores de TG e menores de HDL, e aumento de gordura visceral), foram estatisticamente significativas nas mulheres. Nos homens, as mesmas alterações foram evidenciadas, porém não atingiram significância estatística. A redução do tamanho amostral (I2 homens) poderia ser responsável por este achado. Tal achado está de acordo com a literatura ${ }^{4,6} \mathrm{e}$ provavelmente confere às mulheres deficientes de GH um aumento de incidência de doenças cardiovasculares 4 .

No estudo das correlações das variáveis consideradas, encontramos correlação positiva entre TG e gordura visceral, medida da cintura e gordura visceral, e correlação negativa entre TG e HDL, tanto nos pacientes quanto nos controles. Não encontramos correlação negativa entre HDL e gordura visceral. Alguns dos achados são semeIhantes aos de Snel et al. ${ }^{20}$, que, em 1995, foram os primeiros a comparar a distribuição do tecido adiposo corporal com as concentrações plasmáticas de lipídios. Foram avaliados 12 adultos com DGH comparados com controles pareados para idade, sexo e IMC. A adiposidade visceral correlacionava-se positivamente com os níveis de TG e inversamente com HDL, tanto nos pacientes quanto nos controles e não se encontrava associada a concentrações de CT e LDL.

Analisando separadamente mulheres e homens, encontramos correlação positiva para TG e gordura visceral apenas nas mulheres. Em ambos os sexos, encontramos correlação positiva para gordura visceral e medida da cintura. Tal achado demonstra que a medida da cintura pode ser um método útil para a avaliação de adiposidade visceral. N. Vahl et al. ${ }^{25}$ estudaram 27 deficientes de $\mathrm{GH}$ pareados com controles para idade e sexo. Nos controles, os níveis de CT e TG se correlacionaram positivamente idade, gordura subcutânea e adiposidade abdominal. Nos pacientes, os níveis basais de TG se correlacionaram positivamente com adiposidade subcutânea.

A terapia de reposição de $\mathrm{GH}$ possibilitaria melhora do perfil lipídico, com diminuição dos níveis de $C T$, LDL e Apo B, sem alterações significativas nos níveis de HDL e TG. Com relação à composição corporal, a reposiçãa com GH proporcionaria aumento de massa magra com diminuição de massa gorda e da adiposidade abdominal visceral e, possivelmente, diminuição no risco cardiovascular desses pacientes ${ }^{31,32}$.

\section{Conclusão}

Quando comparamos pacientes adultos deficientes de $\mathrm{GH}$ a um grupo controle, demonstramos que existe nos pacientes aumento da adiposidade visceral e aumento das concentrações de TG com diminuiçãa das concentrações de HDL. As alterações encontradas são mais proeminentes nas mulheres e há correlação positiva entre adiposidade visceral e níveis de TG, sem correlação negativa entre adiposidade visceral e níveis de HDL.

\section{Conflito de interesse: não há.}

\section{SUMMARY}

LIPID PROFILE AND ALTERATION OF BODY COMPOSITION IN GROWTH HORMONE DEFICIENT ADULTS

BACKGROUND. The growth hormone deficiency (GHD) syndrome in adults and the increased associated cardiovascular risk have been extensively studied in recent years. Abnormal body composition with 
excess of visceral adiposity and adverse lipid profile are important features of this syndrome. Abnormallipid profile has been described with increased levels of total cholesterol (C), LDL-cholesterol (LDL-C), triglycerides, decreased levels of HDL-cholesterol (HDL-C) and apolipoproteins abnormalities.

METHODS. Lipid profile and the amount of visceral adipose tissue were studied in 3 I GHD adults compared with a control group of healthy subjects matched for age, gender and body mass index (BMI). Visceral adipose tissue was evaluated by abdominal computed tomography and anthropometric measurements- $\mathrm{BMI}\left(\mathrm{kg} / \mathrm{m}^{2}\right)$ and waist circumference (cm). The lipid profile was studied by measurement of C, LDL-C, HDL-C, triglycerides, apolipoproteins A and B, and Lipoprotein (a).

RESULTS. The GHD adults showed increased visceral adipose tissue (I $\left.56.66 \pm 72.72 \mathrm{vs} .1 / 3.51 \pm 32.97 \mathrm{~cm}^{2}, p=0.049\right)$, higher levels of triglycerides (I $58.58 \pm 80.29$ vs. $97.17 \pm 12.37 \mathrm{mg} / \mathrm{dl} ; p=0.007$ ) and lower HDL-cholesterol (45.4l \pm 13.30 vs. $55.34 \pm 14.31 \mathrm{mg} / \mathrm{dl}$; $p=0.002)$. There were no differences in others aspects oflipid profile and anthropometric measurements.

CONCLUSION. Growth Hormone Deficient adults showed increased visceral adipose tissue, higher levels of triglycerides and lower HDLcholesterol levels. [Rev Assoc Med Bras 2005; 5 I (5): 265-9]

KeY WORDS: Lipids. Body composition. Hypopituitarism. Somatropin.

\section{REFERÊNCIAS}

I. Rosen T, Bengtsson BA. Premature mortality due to cardiovascular disease in hypopituitarism. Lancet 1990;336(I):285-8.

2. Rosen T, Edén S, Larson G, Wilhelmsen L, Bengtsson BA. Cardiovascular risk factors in adults patients with growth hormone deficiency. Acta Endocrinol 1993; I29(3): 195-200.

3. Bullow B, Hagmar L, Mikoczy Z, Nordstrom CH, Erfurth EM. Incresead cerebrovascular mortality in patientes with hypopituitarism. Clin Endocrinol (Copenh) 1997;46(I):75-8I

4. Bullow B, Hagmar L, Eskilsson J, Erfurth EM. Hypopituitary females have a high incidence of cardiovascular morbidity and an increased prevalence of cardiovascular risk factors. J Clin Endocrinol Metab 2000;85(2):574-84.

5. DeBoer H, Blok GJ, Voerman HJ, De Vries PM, Van der Veen EA. Body composition in adult growth hormone deficient men, assessed by anthropometry and bioimpedance analysis. J Clin Endocrinol Metab 1992;75(3):833-7

6. Abdu TA, Neary R, Elhadd TA, Abker M, Clayton RN. Coronary risk in growth hormone deficient hypopituitay adults: incresead predictes risk is due largely to lipid profile abnormalities. Clin Endocrinol (Oxf) 200 I;55(2):209- I 6.

7. Christopher M, Hew FL, Oakley M, Rantzau C, Alford F. Defects of insulin action and skeletal muscle glucose metabolism in growth hormone deficient adults persist after 24 months of recombinant human growth hormone therapy. J Clin Endocrinol Metab 1998,83(5): | 668-8| .

8. Fédou C, Brun J, Raynaud E, Boyer P, Rodier M, De Boisvilliers F, et al. Insulin sensivity and glucose effectiveness measured with the minimal model in adults with GH deficiency. Endocrinol Metab 1996;3(3):99- 104.

9. Johansson JO, Fowelin J, Landin K, Liager I, Bengtsson BA. Growth hormone deficient adults are insulin resistant. Metabolism 1995;44(9): I I 26-9.

10. Conceição FL, Costa e SilvaA, Leal CostaAJ, Vaisman M. Glucagon stimulation test for the diagnosis of $\mathrm{GH}$ deficiency in adults. Endocrinol Invest 2003;26(I I): 1065-70.

I I. Beshyah SA, Freemantle C, Thomas E, Rutherford O, Page B, Murphy M, et al. Abnormal body composition and reduced bone mass in growth hormone deficient hypopituitary adults. Clin Endocrinol (Oxf) I 995; 42(2): I79-89.

12. Jorgensen JO, Vahl N, Hansen TB, Thuesen L, Hagen C, Chistiasen JS. Growth hormone versus placebo treatment for one year in growth hormone deficient adults: increase in exercise capacity and normalization of body composition. Clin Endocrinol (Oxf) 1996;45(6):68 I-8.
13. Jorgensen JOL, Pedersen AS, Thuesen L, Jorgensen J, Moller J, Skakkebaek $\mathrm{NE}$, et al. Long-term growth hormone treatment in growth hormone deficient adults. Acta Endocrinol 199|; | 25(5):449-53.

14. Salomon F, Cuneo RC, Hesp R, Sonksen PH. The effects of treatment with recombinant human growth hormone on body composition and metabolism in adults with growth hormone deficiency. N Eng | Med I 989;32 I (26): I 797-803.

I5. Hew FL, Alford FP, Christopher M. Effects of growth hormone deficiency and therapy in adults on skeletal muscle glucose metabolism, lipid profiles and regional body composition. Endocrinol Metab 1996;3(I):55-60.

16. Pfeifer M, Verhovec R, Zizek B, Prezelj J, Poredos P, Clayton RN. Growth hormone $(\mathrm{GH})$ treatment reverses early atherosclerotic changes in $\mathrm{GH}$ deficient adults. J Clin Endocrinol Metab 1999;84(2):453-7.

17. De Boer H, Blok G, Voerman HJ, Philips M, Schouten JA. Serum lipid levels in growth hormone deficient men. Metabolism 1994:43(2): 199-203.

18. Attanasio AF, Lamberts SW, Matranga AMC, Birkett MA, Bates PC, Valk NK, et al. Adult growth hormone- deficient patients demonstrate heterogeneity between childhood onset and adult onset before and during human $\mathrm{GH}$ treatment. Adult Growth Hormone Deficiency Study Group. J Clin Endocrinol Metab 1997;82(I):82-8.

19. O'Neal DN, Hew FL, Sikaris K, Ward G, Alford F, Best JD. Low density lipoprotein particle size in hypopituitary adults on conventional hormone replacement therapy. J Clin Endocrinol Metab 1996;8 I (7):2448-554.

20. Snel YE, Doerga ME, Brummer RM, Zelissen PM, Kopperchaar HP. Magnetic resonance imaging-assessed adipose tissue and serum lipid and insulin concentrations in growth hormone deficient adults. Effects of growth hormone replacement. Arterioscler Thromb Vasc Biol I 995; I 5( I 0): I 543-8.

21. Austin MA, Breslow JL, Hennekens CH, Buring JE, Willett WC, Krauss RM. Low density lipoprotein subclass patterns and risk of myocardial infarction. JAMA 1988;260(13): 1917-21.

22. Cuneo RC, Salomon F, Wiles CM, Watts GF, Hesp R, Sonksen PH. Growth hormone treatment improves serum lipids and lipoproteins in adults with growth hormone deficiency. Metabolism 1993;42(I 2): I 5 19-23.

23. Al Shoumer KA, Cox KH, Hughes CL, Richmond W, Johnston DG. Fasting and postprandial lipid abnormalities in hypopituitary women receiving conventional replacement therapy. J Clin Endocrinol Metab 1997;82(8): 2653-9.

24. Murray RD, Wieringa GE, Lissett CA, Dargy KH, Smethurst LE, Shalet SM. Low-dose GH replacement therapy on lipoprotein (a) and other lipid parameters in adults with acquired GH deficiency. Results of a double- bind and placebo- controlled trial. Clin Endocrinol (Oxf) 2002;56(4):525-32.

25. Vahl N, Jorgensen JO, Hansen TB, Klausen IB, Jurik AG, Hagen C, et al. The favourable effects of growth hormone $(\mathrm{GH})$ substitution on hypercholesterolaemia in $\mathrm{GH}$ - deficient adults are not associated with concomitant reductions in adiposity. A I 2 month placebo- controlled study. Int J Obes Relat Metab Disord 1998;22(6):529-36.

26. Wajchenberg BL. Subcutaneous and visceral adipose tissue: their relation to the syndrome metabolic. Endocrine Rev 2000;2I (6):697-738.

27. Després JP, Moorjani S, Lupien PJ, Tremblay A, Lupien PJ, Nadeau A, et al. Regional distribution of body fat, plasma lipoproteins and cardiovascular disease. Arteriosclerosis 1990; I 0(4):497-5। I.

28. Pouliot MC, Després JP, Moorjani S, Tremblay A, Lupien PJ, Nadeau A, et al. Computed- tomography measured trunk fat and plasma lipoprotein levels in nonobese women. Metabolism 1989;38( I 2): I 244-50.

29. Johannsson G, Oscarsson J, Rosen T, Wilklund O, Olsson G, Wilhelsem L, et al. Effects of I year of growth hormone therapy on serum lipoproteins levels in growth hormone- deficient adults. Influence of gender and Apo (a) and Apo (e) phenotypes. Arterioscl Thromb Vasc Biol 1995; I 5( I 2):2 I 42-50.

30. Jansson JO, Eden S, Isaksson $\mathrm{O}$. Sexual dimorphism in the control of $\mathrm{GH}$ secretion. Endocr Rev 1985;6(2): I 28-50.

3 I. Jorgensen JOL, Pedersen SA, Thuesen L, Jorgensen J, Ingemann-Hansen T, Skakkebaek NE, et al. Beneficial effects of growth hormone treatment in $\mathrm{GH}$ deficients adults. Lancet I989; I (8649): I 22 | -5.

32. Salomon F, Cuneo R, Hesp R, Sonken PH. The effects of treatment with recombinant human growth hormone on body composition and metabolism in adults with growth hormone deficiency. N Eng J Med | 989;32 | (26): | 797-803.

Artigo recebido: 07/05/04

Aceito para publicação: 22/02/05 